\title{
Articulating Cervical Rib - A Culprit Often Overlooked on Imaging! : Review Article
}

\author{
Pratik S. Itti ${ }^{1}$, Sharad B. Ghatge ${ }^{2}$, Shraddha Somani ${ }^{3}$, Javeriya Fatima ${ }^{4}$ \\ ${ }^{1,3,4}$ Resident, ${ }^{2}$ Associate Professor, \\ Department of Radiology, Grant Government Medical College and Sir JJ Group of Hospitals, Mumbai
}

Corresponding Author: Pratik S. Itti

\begin{abstract}
Background: Cervical rib, though it is seen incidentally in the radiographic examination of chest and cervical spine, a particular subset of it can present with thoracic outlet syndrome. This article mainly focuses on the symptom causation of articulating cervical rib, as compared to non-articulating cervical rib which is often asymptomatic. There are many articles describing a cervical rib, but those highlighting this particular relation between the types of cervical rib and symptom causation are not present in present database. The review of serial radiographs upon correlating clinically, lead us to conclude that articulating variant of cervical rib is majorly symptomatic and caused grave arterial complications. The article consists brief discussions on the types of cervical ribs, thoracic outlet syndrome and role of imaging in diagnosing a cervical rib.

Main Body: We have illustrated five such cases from our tertiary care hospital and highlighted the relationship between the magnitude of symptoms and variations of cervical rib. In compliance with PRISMA guidelines, we screened 33 articles dealing with cervical rib and 13 with thoracic outlet syndrome. Overall, 46 articles were filtered on studying the abstract. Further, 15 more had to be excluded as they were case reports and case series, and finally 31 articles were included in our study. We studied and discussed these articles and research papers in relation to variant anatomy of cervical rib, its types and symptom causation. In our study, also routine sequential chest radiographs were reviewed during the period of January 2020 to January 2021 and those with cervical rib and/or elongated C7 transverse process were included.
\end{abstract}

Conclusion: Thus, to conclude we can state that articulating cervical ribs pose a greater threat and cause arterial occlusion.

Key Words: Cervical rib, thoracic outlet syndrome, subclavian artery

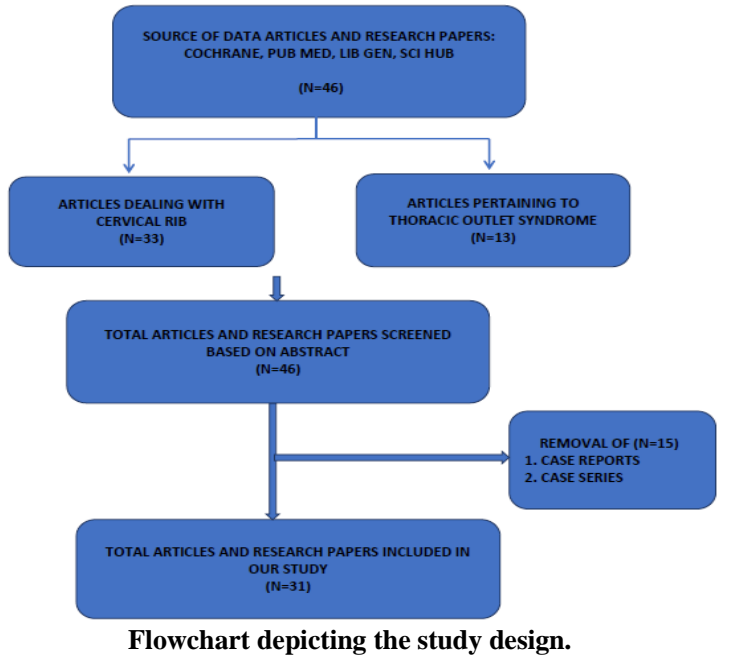

\section{INTRODUCTION}

Cervical rib, though it is seen incidentally in the radiographic examination of chest and cervical spine, a particular subset of it can present with thoracic outlet syndrome.

Thoracic outlet syndrome is a condition in which there is compression of the neurovascular bundle in the compactly packed passage from lower neck to the axillary region. This is most commonly ascertained to repetitive strain and trauma and less likely to anatomical bony variations and tumors. Patients often present with 
chronic symptoms of pain, tingling and numbness along the upper limb, thus neurological bundle being the most frequently affected. Venous compression would cause bluish discoloration and swelling, while arterial compression would result in pulselessness, dry-cold limb and gangrene at a later stage. Anatomical variations being our focus point in this article refers to cervical rib - its types and articulations, elongated transverse processes of C7. Patients often get treated for these symptoms either as cervical spondylosis or shoulder related issues thus overlooking their silent presence in the routine chest radiography. We have illustrated five such cases from our tertiary care hospital and highlighted the relationship between the magnitude of symptoms and variations of cervical rib.

\section{DISCUSSION \\ Illustrative cases: \\ Case 1:}

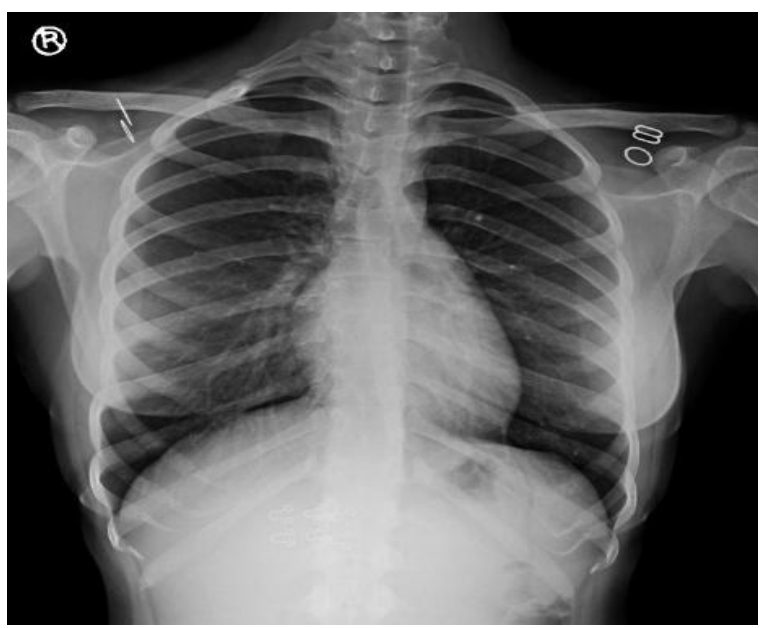

Fig. 1. Chest radiograph of middle aged female showing complete bony right cervical rib seen articulating with the first rib.

35 year old woman presented with pain, numbness over right upper limb and right supraclavicular swelling since many years. On examination, the swelling [10] felt bony hard on palpation. Chest radiograph revealed right sided complete bony cervical rib articulating with the first rib. On doppler study, the subclavian artery was seen compressed by the cervical rib as it passed between the cervical rib and first rib with significant post stenotic dilatation. This was further confirmed on CT angiography. However, no thrombus or reduced flow in the distal arterial system was appreciated at present (Fig. 1).

Case 2: 48 year old man presented with cold right upper limb with color changes at finger tips since few days. On routine chest imaging, radiograph revealed elongated bilateral C7 transverse processes and a complete bony right cervical rib forming pseudo-joint with the first rib. Doppler study revealed complete acute thrombosis of right subclavian artery at the site of compression due to cervical rib. Axillary, brachial, ulnar and radial arteries showed reduced flow with scanty collaterals (Fig 2).

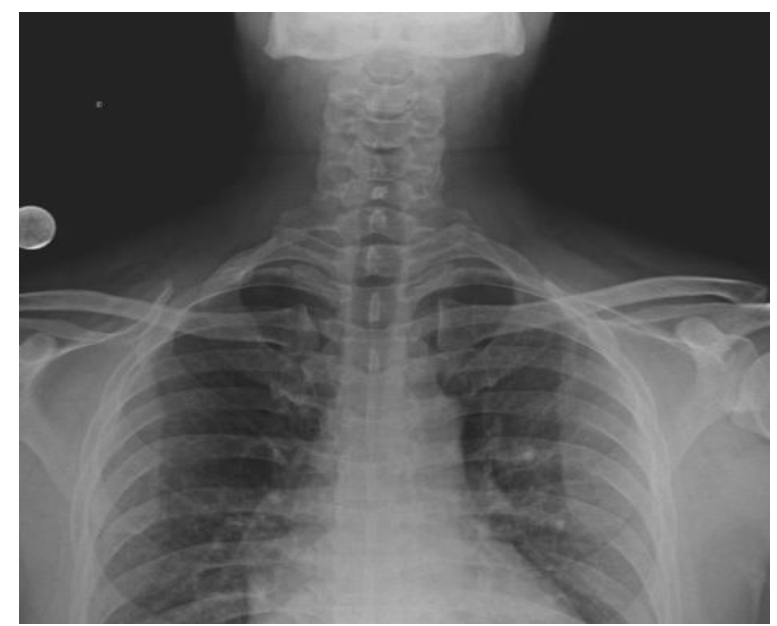

Fig. 2. Chest radiograph of 48 year old male showing complete bony right cervical rib seen articulating with the first rib and elongated bilateral $\mathrm{C} 7$ transverse processes.

\section{Case 3:}

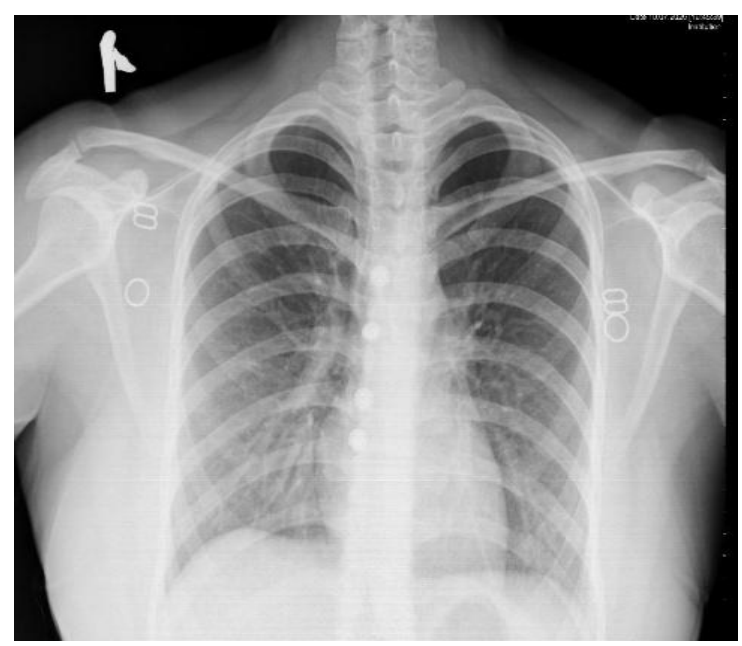

Fig. 3. Chest radiograph of 25 year old female showing elongated bilateral $\mathrm{C} 7$ transverse processes. 
25 year old student complained of occasional numbness and tingling sensation along the ulnar aspect of both upper limbs. Chest radiograph revealed, elongated bilateral C7 transverse processes [1]. She underwent magnetic resonance imaging of cervical spine too, which turned out to be normal. Thus, attributing the elongated transverse processes as a cause to her symptoms (Fig. 3).
Case 4: Adolescent man presented with left upper limb pain and numbness since 15 days. On examination, left radial pulse was absent. Chest radiograph revealed incomplete left first rib. On selective digital subtraction angiography of left subclavian artery, the middle third of the artery was seen compressed by the incomplete left first rib causing turbulent flow and embolic occlusion of distal radial and ulnar arteries (Fig. 4).
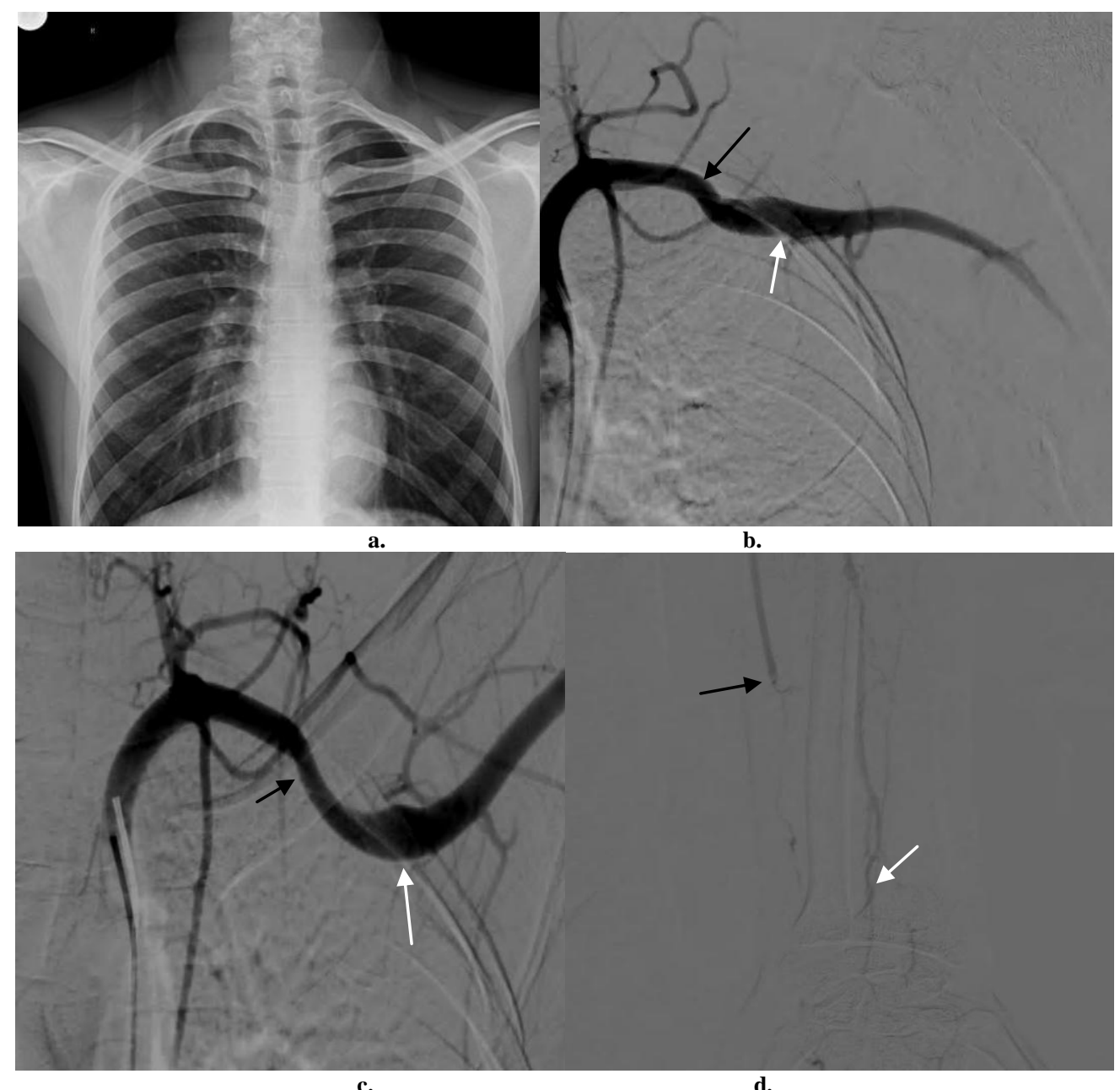

Fig. 4. Chest radiograph of 44 year old man (a) showing incomplete bony left cervical rib. Digital subtraction angiography image of the subclavian artery (b) showing stenosis (black arrow) secondary to partial lumen occluding thrombosis in the second/mid segment of subclavian artery with post stenotic dilatation (white arrow). On Allen's maneuver (c) (abduction and external rotation of the arm), the stenosis becomes exaggerated. Digital subtraction image of brachial artery (d) showing no flow in distal radial (white arrow) and ulnar (black arrow) arteries of the patient.

Case 5: A middle aged woman presented to the emergency room with cold left upper limb and associated numbness since few days. Chest radiograph showed complete bony left cervical rib articulating with the first rib and incomplete bony right cervical rib. CT upper limb angiogram revealed focal stenosis/pinching of middle third of subclavian artery between the cervical rib and clavicle with associated lumen occluding thrombus formation. Patient underwent thrombectomy and cervical rib excision after which the flow was restored to left upper limb (Fig. 5). 

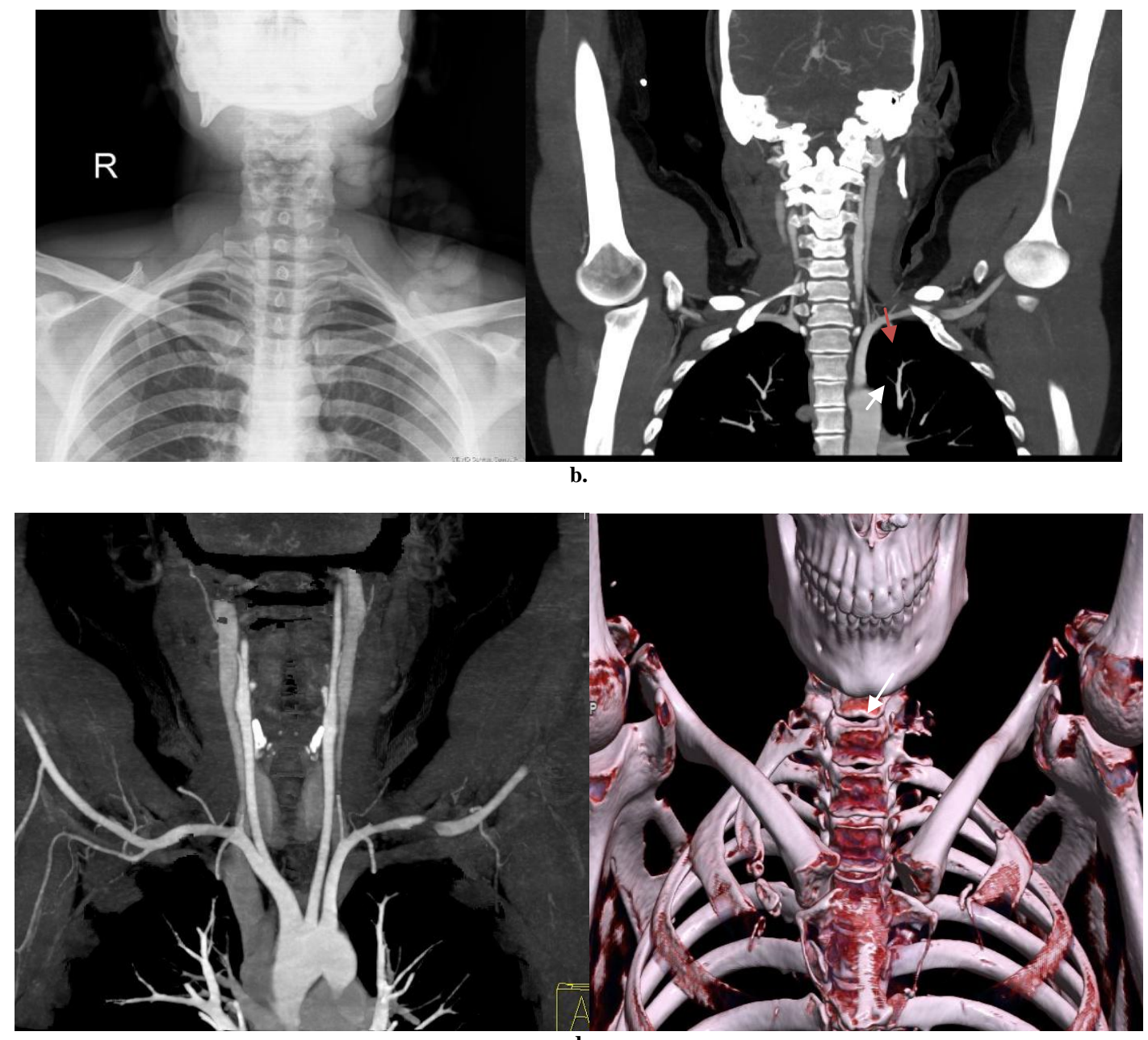

d.

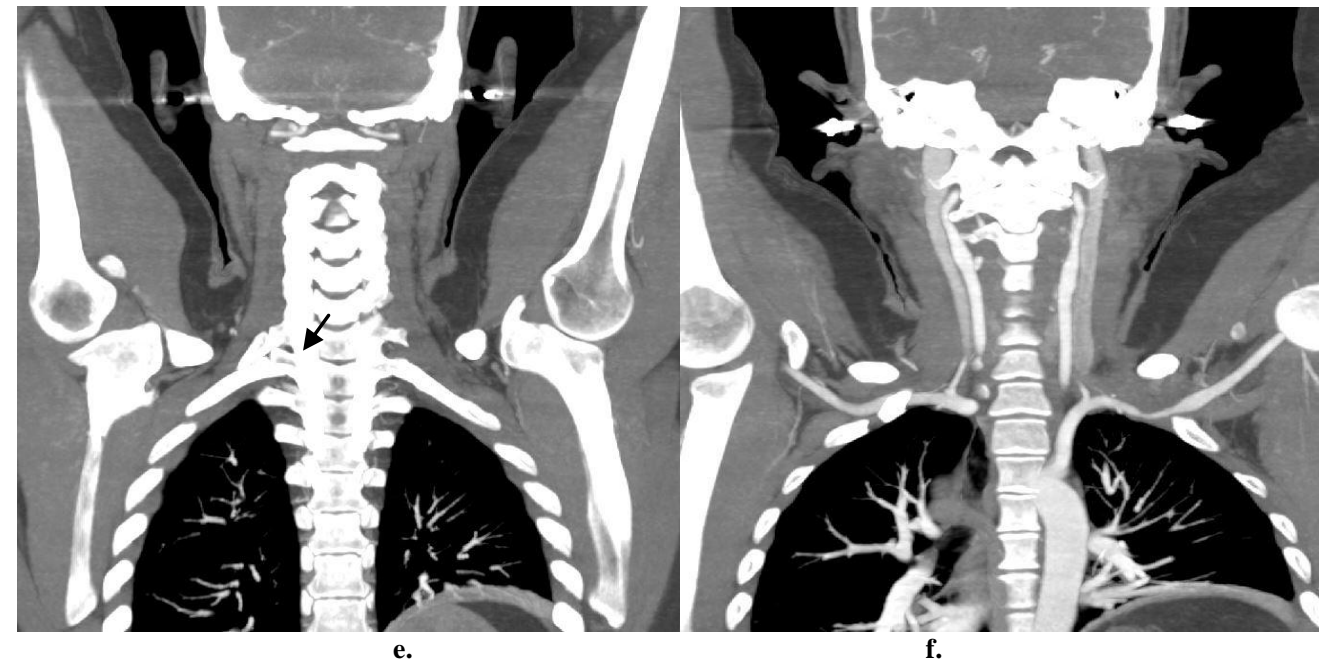

Fig. 5. Chest radiograph (a) of a middle aged woman showing complete bony left cervical rib articulating with the first rib and incomplete bony right cervical rib. CT upper limb angiogram (b) revealed focal stenosis/pinching of middle third of left subclavian artery between the cervical rib (white arrow) and clavicle (orange arrow). CT angiogram (c) showing near complete thrombosis of mid third of subclavian artery at the site of stenosis. Patient underwent thrombectomy and cervical rib excision; hence the left cervical rib is not visualized (white arrows) in post-operative CT (e) and 3D reconstructed image of the same (d). The flow is restored across the subclavian artery as seen in post-operative $\mathrm{CT}$ angiogram (f).

\section{CONCLUSIONS}

The interpretation based on the observations made and by reviewing the existing literature tells that the articulating cervical rib, which is the major culprit for severe symptom causation. 
To our knowledge the types of cervical rib can be discussed as two groups [7]:

I. Based on its composition II. Based on its structure;

The first classification would include (based on composition):

1. Completely bony

2. Completely fibrous

3. Combined

The second classification would include (based on structure):

A. Complete (Articulating)

B. Incomplete (non articulating)

i. With fibrous band

ii. Without fibrous band

C. Elongated $\mathrm{C} 7$ transverse process

D. Bony swelling

As per the experience in our tertiary care hospital it was seen that patients with a complete bony articulating cervical rib had more serious and pre dominantly arterial pathologies. The mid part of subclavian artery in the scalene triangle gets pinched either between the cervical and first rib or between the cervical rib and clavicle. Also, the artery can get compressed by the lower border of cervical rib. This results in luminal narrowing at the site of compression with post stenotic dilatation resulting in turbulent flow causing endothelial injury and finally causing thrombosis at that site or distal embolic occlusion (Fig. 6).

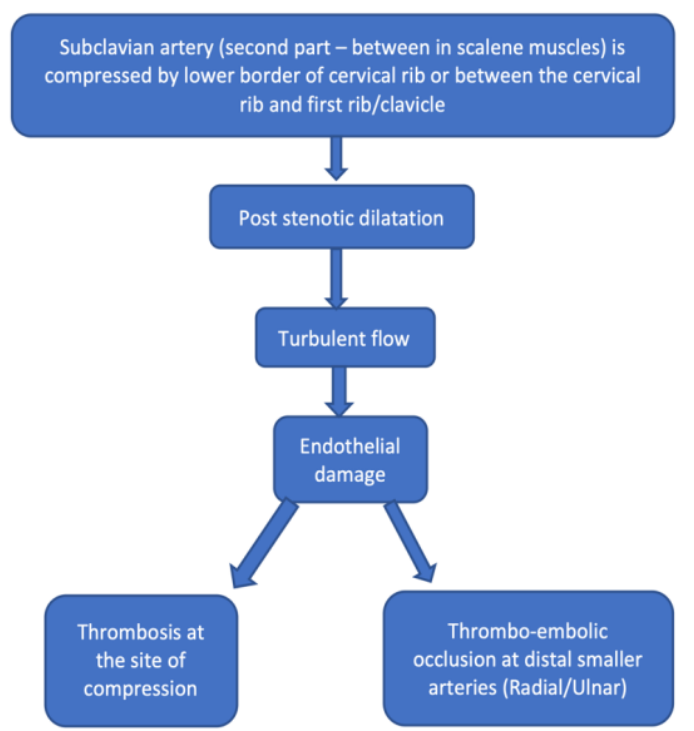

Fig. 6. Flow chart showing brief pathogenesis of cervical rib causing upper limb arterial thromboembolism.
Other bony variants causing TOS [7]:

1. Anomalous first rib: Developmental variation resulting in floating first rib with/without fibrous band.

\section{Abnormal first rib/clavicle:} Hypertrophic callus secondary to old fracture or bony tumors involving the first rib or clavicle.

Thoracic outlet syndrome [12] refers to compression of the neurovascular bundle within the thoracic outlet [9]. Based on the structure being compressed it can be symptomatically and pathologically classified into:

Neurogenic TOS: Compression brachial plexal cords or sympathetic chain [3]. This is by far the most common variety where in the patient presents with tingling, numbness along the ulnar border of upper arm and muscle weakness in more severe type. MRI would be the modality of choice in demonstrating the cause. MR imaging can be done in neutral adduction position of the upper limb and then with the limb abducted to the degree which the machine bore would allow. It is observed that the compression of the structures increases on abducting the arm.

Venous TOS: The subclavian vein gets compressed causing symptoms of central venous occlusion i.e. Swelling of upper limb and anterior chest wall with dilated superficial veins. USG doppler and CT venogram would aid our diagnosis. Catheter venogram with sos treatment in the form of stenting or thrombectomy is also gaining widespread popularity. The root treatment would definitely be excision of the cervical rib.

Arterial TOS: Arterial stenosis and thrombosis is witnessed with symptoms that of claudication and gangrene at a later stage [14]. CT angiogram is considered the ideal modality as we can demonstrate both the bony culprit and the arterial compression/ thrombosis. DSA with selective catheterization of subclavian origin is gold 
standard. We propose the technique of taking images in neutral position where in the arm is adducted and in abducted position with 90 degree elbow flexion and patients head turned to the opposite side (Allen's maneuver). Now, during the Allen's maneuver the artery is seen getting compressed more and the distal radial and ulnar arteries fill up slowly thus further compromising the blood supply.

\section{Role of imaging -}

Most of the cases are asymptomatic and are hence picked up on our routine chest radiograph and cervical spine imaging. $\mathrm{X}$ ray being a basic modality $[7,11]$ is the first step in the diagnosis. Bony variants can be seen on a radiograph requiring no special position. However, the comment on articulation and fibrous bands in incomplete ribs is a drawback [6]. Thus, requiring higher modalities for complete evaluation.

Computed tomography (CT) aids in our complete diagnosis of the cervical rib [8], its type, articulation and the presence of the fibrous band. Added contrast scans, such as angiography further show us the site of compression of the subclavian vessels. Thrombus if present, can also be demonstrated in the same. The compression being aggravated on abduction position of the arm can be demonstrated by taking a control image with the arm in adduction and another image with the arm abducted [4, 15]. This also aids the surgeons before they go on to resect the cervical rib.

Magnetic resonance (MR) imaging adds to our diagnosis [5,13], especially if we are dealing with a neurogenic TOS. It further improves the visualization of soft tissue (fibrous band) and its nature with the neurovascular bundle. MR imaging [16] is not routinely used in cases of cervical rib, however the conspicuous fibrous band with incomplete ribs and their relation to the brachial plexus and sympathetic chain is best visualized on MR. MR with vessel wall imaging tells us the nature of arterial wall and its condition which helps the vascular surgeons if they are planning on bypass graft procedures. Images can be obtained with the arm in adduction and abduction to demonstrate the aggravation of compression in relation to the arm position.

Digital subtraction angiography (DSA) stays the gold standard modality in case of arterial TOS. It gives us a distinct picture on the location of stenosis/ thrombosis with its hemodynamic significance. As per our knowledge, there is no literature describing angiography with Allen's maneuver. With the patients arm in abduction, external rotation and 90 degree flexion at the elbow, the arterial compression becomes more prominent. Furthermore, we can go on and treat the thrombosis by extracting it with a Fogarty's balloon or place a stent in case of stenosis and salvage the limb followed by resection of the cervical rib by the surgeons.

To conclude, it is now evident that complete bony cervical ribs that articulate either with the first rib or the clavicle pose a greater threat. They are prone to cause arterial stenosis and thrombosis. Prophylactic resection of such ribs can be considered in order to prevent the ordeal of salvaging the limb in the symptomatic period or amputating the arm in untimely diagnosed patients.

\section{List of abbreviations:}

CT: Computed tomography

MRI: Magnetic Resonance Imaging

TOS: Thoracic Outlet Syndrome

USG: Ultrasonography

\section{Acknowledgement: None}

\section{Conflict of Interest: None}

\section{Source of Funding: None}

\section{REFERENCES}

1. Bokhari RF, Al-Sayyad MJ, Baeesa SS. Prevalence of cervical ribs and elongated transverse processes in Saudi Arabia. Saudi Med J. 2012 Jan 1;33(1):66-9.

2. Cubuk R, Tasali N, Arslan G, Atasoy M. Arterial compression in a young adult due to the presence of a cervical rib: CT and CT 
angiographic findings. The Eurasian journal of medicine. 2010 Apr;42(1):46.

3. Dashti G, Ghasemi N. Evaluation of neurovascular complication of cervical ribs.

4. Davran R, Bayarogullari H, Atci N, Kayali A, Ozturk F, Burakgazi G. Congenital abnormalities of the ribs: evaluation with multidetector computed tomography. J Pakistan Med Assoc. 2017 Feb 1;67:178-86.

5. Gupta A, Gupta DP, Saxena DK, Gupta RP. Cervical rib: It's prevalence in Indian population around Lucknow (up). Journal of Anatomical Society of India. 2012 Dec 1;61(2):189-91.

6. Keating DR, Amberg JR. A source of potential error in the roentgen diagnosis of cervical ribs. Radiology. 1954 May;62(5): 688-94.

7. Kurihara Y, Yakushiji YK, Matsumoto J, Ishikawa $\mathrm{T}$, Hirata $\mathrm{K}$. The ribs: anatomic and radiologic considerations. Radiographics. 1999 Jan;19(1):105-19.

8. Newmark III H, Cassidy TD. Cervical rib diagnosis by computerized tomography. Computerized radiology. 1986 Jul 1;10(4):171-3.

9. Ozturk S, Yildirim H, Kaplan M. Is the existence of cervical rib an advantage for C7 posterior stabilization?. Journal of craniovertebral junction \& spine. 2016 Oct;7(4):201.

10. Roy S, Jain N, Narang E, Singh J. Cervical Rib: A Rare Differential of a Supraclavicular Mass. Ear, Nose \& Throat Journal. 2020 Aug 14:0145561320950495.
11. Salam A, Ahmed MU, Kohistani TA. Radiographic evaluation of cervical spine. Rawal Medical Journal. 2010;35(2):152-5.

12. Selmonosky CA, Silva PR. Congenital abnormalities, cervical ribs, and related bony abnormalities. InThoracic outlet syndrome 2013 (pp. 571-577). Springer, London.

13. Singh VK, Jeyaseelan L, Kyriacou S, Ghosh S, Sinisi M, Fox M. Diagnostic value of magnetic resonance imaging in thoracic outlet syndrome. Journal of Orthopaedic Surgery. 2014 Aug;22(2):228-31.

14. Sharma P, Rasheed I, Ansari MA, Gurung GS, Chataut SP. Cervical rib causing thrombosis of subclavian artery. Journal of the Nepal Medical Association. 2010 Apr $1 ; 49(178)$.

15. Viertel VG, Intrapiromkul J, Maluf F, Patel NV, Zheng W, Alluwaimi F, Walden MJ, Belzberg A, Yousem DM. Cervical ribs: a common variant overlooked in CT imaging. American Journal of Neuroradiology. 2012 Dec 1;33(11):2191-4

16. Walden MJ, Adin ME, Visagan R, Viertel VG, Intrapiromkul J, Maluf F, Patel NV, Alluwaimi F, Lin D, Yousem DM. Cervical ribs: identification on MRI and clinical relevance. Clinical imaging. 2013 Sep 1;37(5):938-41.

How to cite this article: Itti PS, Ghatge SB, Somani S et.al. Articulating cervical rib - a culprit often overlooked on imaging! : review article. Int J Health Sci Res. 2021; 11(9): 230236. DOI: https://doi.org/10.52403/ijhsr. 20210935 